\title{
APPROXIMATIONS AND REPRESENTATIONS FOR FOURIER TRANSFORMS
}

\author{
BY \\ RAOUF DOSS
}

\begin{abstract}
G$ is a locally compact abelian group with dual $\Gamma$. If $p(\gamma)=\sum_{1}^{N} a_{n}\left(x_{n}, \gamma\right)$ is a trigonometric polynomial, its capacity, by definition is $\sum\left|a_{n}\right|$. The main theorem is: Let $\varphi$ be a measurable function defined on the measurable subset $\Lambda$ of $\Gamma$. If $\varphi$ can be approximated on finite sets in $\Lambda$ by trigonometric polynomials of capacity at most $C$ (constant), then $\varphi=\hat{\mu}$, locally almost everywhere on $\Lambda$, where $\mu$ is a regular bounded measure on $G$ and $\|\mu\| \leqq C$.
\end{abstract}

In this paper $G$ is a locally compact abelian group with dual $\Gamma$. The set of bounded regular measures on $G$ will be denoted $M(G)$. If $\mu \in M(G)$ its transform $\hat{\mu}$ is defined by

$$
\hat{\mu}(\gamma)=\int_{G}(x,-\gamma) d \mu(x), \quad \gamma \in \Gamma .
$$

DEFINITION. If $p(\gamma)=\sum_{1}^{N} a_{n}\left(x_{n}, \gamma\right)$ is a trigonometric polynomial on $\Gamma$, its capacity, by definition, is $\sum_{1}^{N}\left|a_{n}\right|$. If $s(\gamma)=\sum_{1}^{\infty} a_{n}\left(x_{n}, \gamma\right)$ with $\sum_{1}^{\infty}\left|a_{n}\right|=C<\infty$, then $s(\gamma)$ will be called a trigonometric series with capacity $C$.

Now if $\varphi$ is any continuous function on $\Gamma$, then $\varphi$ can be uniformly approximated on compact sets in $\Gamma$ by trigonometric polynomials (Stone-Weierstrass theorem). In general the capacities of these polynomials will be unbounded. If we demand that these capacities be bounded by a fixed constant $C$ we get a characterization of the transform of a measure. We shall prove

Proposition 1. Let $\varphi$ be a function defined on $\Gamma$. In order that $\varphi=\hat{\mu}$ for some $\mu \in M(G)$ it is necessary and sufficient that there exists a constant $C$ such that $\varphi$ can be uniformly approximated on any compact set in $\Gamma$ by trigonometric polynomials of capacity at most $C$.

This approximation property can be strengthened to a representation property. In fact,

Proposition 1'. Let $\varphi$ be a function defined on $\Gamma$. In order that $\varphi=\hat{\mu}$ for some $\mu \in M(G)$ it is necessary and sufficient that there exists a constant $C$ such that for any compact set $\Lambda$ in $\Gamma, \varphi$ is equal on $\Lambda$ to a trigonometric series of capacity at most $C$.

Received by the editors April 14, 1969 and, in revised form, February 13, 1970.

AMS 1969 subject classifications. Primary 4250, 4252.

Key words and phrases. Locally compact abelian group, Fourier-Stieltjes transform of a measure, trigonometric polynomials, approximation.

Copyright (C) 1971, American Mathematical Society 
These propositions are not entirely new for they are equivalent to known results, stated differently (see below).

A function $\varphi$ with the above approximation or representation property is automatically continuous. It is an important theorem of Bochner [2] and Eberlein [5] that the continuity (or even measurability) of $\varphi$, combined with the approximation property on finite [instead of compact] sets in $\Gamma$, implies that $\varphi=\hat{\mu}$ (almost everywhere in case of measurability of $\varphi$ ), where $\mu \in M(G)$. In fact, the BochnerEberlein theorem may be given the form:

THEOREM B-E. Let $\varphi$ be continuous (resp. measurable) on $\Gamma$. If $\varphi$ can be approximated on any finite set in $\Gamma$ by trigonometric polynomials of capacity at most $C$, then $\varphi=\hat{\mu}$ (resp. locally almost everywhere) where $\mu \in M(G)$ and $\|\mu\| \leqq C$.

Our main result is an analogous theorem valid for restrictions to a measurable subset $\Lambda$ of $\Gamma$. Namely,

TheOREM. Assume $\varphi$ is measurable on the measurable set $\Lambda$ in $\Gamma$ and that $\varphi$ is approximable on finite sets in $\Lambda$ by trigonometric polynomials with capacity at most $C$, then $\varphi=\hat{\mu}$ locally almost everywhere on $\Lambda$, where $\mu \in M(G)$ and $\|\mu\| \leqq C$.

Particular cases of this theorem are due to Bochner [2]: $\Gamma=R, \Lambda=R$; to Krein (cf. [1, pp. 154-159]): $\Gamma=R, \Lambda=$ an interval; to Eberlein [5]: $\Lambda=\Gamma$; and to Rosenthal [7]: $\Gamma=R$ although their statements are expressed somewhat differently but equivalently. (See below.)

In the final part of the paper we restate in a new form, the result appearing in [4], that the transform of an integrable function lives mostly on sompact sets, while the transform of a singular measure is scattered all over $\Gamma$.

The proof of Propositions 1 and $1^{\prime}$ is based on the following.

Proposition 2. Let $\varphi$ be a function defined on a subset $\Lambda$ of $\Gamma$. Then the following two statements are equivalent:

(A) $\varphi$ is approximable on finite sets in $\Lambda$ by polynomials with capacity at most $C$.

(B) If $q(x)=\sum_{1}^{M} b_{m}\left(x, \gamma_{m}\right)$ with $\gamma_{m} \in \Lambda$ and $\|q\|_{\infty} \leqq 1$ then $\left|\sum b_{m} \varphi\left(\gamma_{m}\right)\right| \leqq C$.

Proof. Assume (A) holds. Let $q(x)=\sum_{1}^{M} b_{m}\left(x, \gamma_{m}\right)$, with $\gamma_{m} \in \Lambda$ and $\|q\|_{\infty} \leqq 1$; $\varepsilon>0$ being given, there is, by hypothesis, a polynomial $p(\gamma)=\sum a_{n}\left(x_{n}, \gamma\right)$ with $\sum\left|a_{n}\right| \leqq C$ such that

Then

$$
\left|\varphi\left(\gamma_{m}\right)-p\left(\gamma_{m}\right)\right| \leqq \varepsilon / \sum_{k}\left|b_{k}\right|, \quad m=1, \ldots, M .
$$

But

$$
\left|\sum b_{m} \varphi\left(\gamma_{m}\right)-\sum b_{m} p\left(\gamma_{m}\right)\right| \leqq \varepsilon .
$$

Hence

$$
\left|\sum_{m} b_{m} p\left(\gamma_{m}\right)\right|=\left|\sum_{n} a_{n} q\left(x_{n}\right)\right| \leqq \sum\left|a_{n}\right| \leqq C .
$$

$$
\left|\sum b_{m} \varphi\left(\gamma_{m}\right)\right| \leqq C+\varepsilon .
$$

$\varepsilon$ being arbitrary, statement (B) holds. 
Conversely, assume (B) holds.

Going to the Bohr compactification $\bar{G}$ of $G$, using the Hahn-Banach extension theorem and the Riesz representation theorem, we see that there is a measure $\mu \in M(\bar{G})$, with $\|\mu\| \leqq C$, whose transform $\hat{\mu}$ is equal to $\varphi$ on $\Lambda$.

Let $\left\{\gamma_{1}, \ldots, \gamma_{M}\right\}$ be a finite subset of $\Lambda$. Let $\varepsilon>0$ be given. There is a finite, disjoint, not necessarily open covering $W_{1}, \ldots, W_{N}$ of the compact group $\bar{G}$ such that

$$
\left|\left(\bar{x}, \gamma_{m}\right)-\left(\bar{x}^{\prime}, \gamma_{m}\right)\right|<\varepsilon, \quad m=1, \ldots, M,
$$

provided $\bar{x}, \bar{x}^{\prime} \in W_{n}, n=1, \ldots, N$.

Choose some $\bar{x}_{n} \in W_{n}, n=1, \ldots, N$, and put

$$
\begin{aligned}
a_{n} & =\mu\left(W_{n}\right), \\
\bar{p}(\gamma) & =\sum_{n} a_{n}\left(\bar{x}_{n},-\gamma\right) .
\end{aligned}
$$

Then

$$
\sum\left|a_{n}\right|=\sum\left|\mu\left(W_{n}\right)\right| \leqq\|\mu\| \leqq C .
$$

We have

$$
\hat{\mu}\left(\gamma_{m}\right)=\sum_{n} \int_{W_{n}}\left(\bar{x},-\gamma_{m}\right) d \mu(\bar{x}), \quad m=1, \ldots, M .
$$

If $\bar{x} \in W_{n}$ then $\left|\left(\bar{x}, \gamma_{m}\right)-\left(\bar{x}_{n}, \gamma_{m}\right)\right|<\varepsilon$. Therefore

$$
\left|\int_{W_{n}}\left(\bar{x},-\gamma_{m}\right) d \mu(\bar{x})-\int_{W_{n}}\left(\bar{x}_{n},-\gamma_{m}\right) d \mu(\bar{x})\right|<\varepsilon|\mu|\left(W_{n}\right) .
$$

This is

$$
\left|\int_{W_{n}}\left(\bar{x},-\gamma_{m}\right) d \mu(\bar{x})-a_{n}\left(\bar{x}_{n},-\gamma_{m}\right)\right|<\varepsilon|\mu|\left(W_{n}\right) .
$$

We conclude, from (1) and (2), for $m=1, \ldots, M$,

$$
\left|\hat{\mu}\left(\gamma_{m}\right)-\bar{p}\left(\gamma_{m}\right)\right|<\varepsilon \sum_{n}|\mu|\left(W_{n}\right) \leqq \varepsilon\|\mu\| \leqq \varepsilon C .
$$

Finally, since $G$ is dense in $\bar{G}$ we can choose $x_{n} \in G$ such that

$$
\left|\left(x_{n}, \gamma_{m}\right)-\left(\bar{x}_{n}, \gamma_{m}\right)\right| \leqq \varepsilon, \quad m=1, \ldots, M .
$$

Put $p(\gamma)=\sum_{n} a_{n}\left(x_{n},-\gamma\right)$. Then

$$
\left|\hat{\mu}\left(\gamma_{m}\right)-p\left(\gamma_{m}\right)\right|<2 \varepsilon C, \quad m=1, \ldots, M .
$$

$\varepsilon$ being arbitrary, property (A) holds.

Proposition 2 is now proved.

Proposition 2 shows that our statement of Theorem B-E is equivalent to the original statement of the Bochner-Eberlein theorem. 
Also the sufficiency of the conditions appearing in Propositions 1 and $1^{\prime}$ is a consequence of the sufficiency of the weaker condition appearing in Theorem B-E.

There remains only to show the necessity of the condition in Proposition 1'. But this is precisely a theorem of $\mathbf{K}$. de Leeuw and C. Herz ([3, Theorem 1]; take $G_{1}=G, G_{2}=\bar{G}$, the Bohr compactification of $G$ ).

We now go to the proof of our main theorem.

LEMMA 1. Let $G_{1}$ be a locally compact abelian group of the form $G_{1}=R^{a} \times T^{b} \times D$ where $a, b$ are nonnegative integers and $D$ a discrete group. Let $V_{0}$ be a neighborhood of 0 in $G_{1}$, which is a direct product of compact symmetric neighborhoods of 0 in the factors $R, T$ occurring in $G_{1}$ and of the neighborhood $\{0\}$ of 0 in $D$. Then to any compact set $K$ containing $V_{0}+V_{0}$ we can associate a function $u$ on $G_{1}$ such that

(1) $u \geqq 0$,

(2) $u$ vanishes outside $V_{0}+V_{0}$,

(3) $\int_{G_{1}} u(x) d x=1$,

(4) $u(x)=\sum b_{n}\left(x, \gamma_{n}\right)$ for $x \in K$,

(5) $\sum\left|b_{n}\right| \leqq m_{1}\left(V_{0}\right)^{-1}$,

(6) $\int_{K+x_{0}}\left|\sum b_{n}\left(x, \gamma_{n}\right)\right| d x \leqq 1$ for every $x_{0} \in G_{1}$, where $m_{1}$ is Haar measure on $G_{1}$.

Proof. Case (i). $G_{1}=T$. Let $f$ be the function in $L^{2}\left(G_{1}\right)$ equal to $m_{1}\left(V_{0}\right)^{-1} X_{V_{0}}$ where $X_{V_{0}}$ is the characteristic function of $V_{0}$. Then in $L^{2}(T)$

$$
f(x)=\sum a_{n}\left(x, \gamma_{n}\right), \quad \gamma_{n} \in Z,
$$

where $\sum\left|a_{n}\right|^{2}=\|f\|_{2}^{2}=m_{1}\left(V_{0}\right)^{-1}$. Put $u=f * f$. Since $f$ is nonnegative and symmetric we have

$$
u(x)=\sum b_{n}\left(x, \gamma_{n}\right) \geqq 0
$$

with

$$
\sum\left|b_{n}\right|=\sum\left|a_{n}\right|^{2}=m_{1}\left(V_{0}\right)^{-1}
$$

and $u$ vanishes outside the set $V_{0}+V_{0}$. Also, by Fubini

$$
\int_{G_{1}} u(x) d x=\int_{G_{1}} \int_{G_{1}} f(y) f(x-y) d y d x=1 .
$$

Finally

$$
\int_{K+x_{0}}\left|\sum b_{n}\left(x, \gamma_{n}\right)\right| d x \leqq \int_{G_{1}} u(x) d x=1 .
$$

Case (ii). $G_{1}=R$. Assume that the compact set $K$ is interior to the interval $(-N, N]$ which may be identified with $T$. Define the function $u$ as above:

$$
\begin{array}{ll}
u(x)=\sum b_{n}\left(x, \gamma_{n}\right), & x \in(-N,+N], \\
u(x)=0, & x \notin(-N,+N],
\end{array}
$$


where now $\left(x, \gamma_{n}\right)$ has period $2 N$. Then $\sum b_{n}\left(x, \gamma_{n}\right)$ has period $2 N$ and (6) follows.

Case (iii). $G_{1}=D$ discrete. Here $V_{0}=\{0\}$. Then $m_{1}\left(V_{0}\right)=1$ and

$$
\int_{K+x_{0}}\left|\sum b_{n}\left(x, \gamma_{n}\right)\right| d x=\sum_{x \in K+x_{0}}\left|\sum b_{n}\left(x, \gamma_{n}\right)\right| \text {. }
$$

Let $\bar{G}_{1}$ be the Bohr compactification of $G_{1}$, the dual of $\Gamma_{1}$ made discrete.

We can find a neighborhood $W_{1}$ of 0 in $\bar{G}_{1}$ which meets the finite set $K-K$ in just the point 0 . Let $W_{2}$ be a neighborhood of 0 in $\bar{G}_{1}$ such that $W_{2}-W_{2} \subset W_{1}$. Then

(*) for $x_{0} \in G_{1}$, the set $K+x_{0}$ meets $W_{2}$ in one point at most.

For, assume $k_{1}, k_{2} \in K ; k_{1}+x_{0}, k_{2}+x_{0} \in W_{2}$. Then $k_{1}-k_{2} \in W_{2}-W_{2} \subset W_{1}$ and therefore $k_{1}-k_{2}=0$.

Let $W_{3}$ be a compact symmetric neighborhood of 0 in $\bar{G}_{1}$ such that $W_{3}+W_{3} \subset$ $W_{2}$. Let $F$ be the function in $L^{2}\left(\bar{G}_{1}\right)$ equal to $\bar{m}_{1}\left(W_{3}\right)^{-1 / 2} X_{W_{3}}$ where $\bar{m}_{1}$ is Haar measure in $\bar{G}_{1}$. Then, in $L^{2}\left(\bar{G}_{1}\right)$,

$$
F(\bar{x})=\sum a_{n}\left(\bar{x}, \gamma_{n}\right), \quad \bar{x} \in \bar{G}_{1}, \gamma_{n} \in \Gamma_{1},
$$

where $\sum\left|a_{n}\right|^{2}=\|F\|_{2}^{2}=1$. Put $U=F * F$. Then

$$
U(\bar{x})=\sum b_{n}\left(\bar{x}, \gamma_{n}\right) \geqq 0, \quad \bar{x} \in \bar{G}_{1},
$$

with $\sum b_{n}=\sum\left|b_{n}\right|=\sum\left|a_{n}\right|^{2}=1$ and $U$ vanishes outside $W_{3}+W_{3}$; in particular, $U$ vanishes outside $W_{2}$. Since, by (*), $K$ meets $W_{2}$ in just the point 0 , then $U(x)=0$ for $x \in K, x \neq 0$. Also $U(0)=1$. Put

$$
u(0)=1, u(x)=0 \text { for } x \in G_{1}, x \neq 0 .
$$

Then conditions (1), (2), (3) are satisfied and

$$
u(x)=\sum b_{n}\left(x, \gamma_{n}\right)=U(x) \text { for } x \in K .
$$

Finally, by $(*)$, at most one term in the sum

$$
\sum_{x \in K+x_{0}}\left|\sum b_{n}\left(x, \gamma_{n}\right)\right|
$$

is different from 0 and this term is at most 1 .

General case. Let $G_{1}$ be the finite direct product of the groups $G_{\alpha}$. If $K$ is compact in $G_{1}$, then $K$ is contained in a direct product $\Pi K_{\alpha}$, where $K_{\alpha}$ is compact in $G_{\alpha}$. Take $u$ to be the product of the $u$ 's constructed for each $G_{\alpha}$.

LeMMA 2. Let $G$ be any locally compact abelian group with dual $\Gamma$. Let $\varphi, f$ be two bounded measurable functions with compact support $\Lambda_{0}$ in $\Gamma$ and let $\varepsilon>0$ be given. Then, for a certain polynomial $p(x)=\sum_{1}^{N} b_{n}\left(x, \gamma_{n}\right)$, we have

$$
\int_{\Lambda_{0}}\left|\sum_{1}^{N} b_{n} \varphi\left(\gamma+\gamma_{n}\right)-\int_{\Gamma} \varphi\right| d \gamma<\varepsilon,
$$


and the function on $\Lambda_{0}$

$$
\sup _{y \in G}\left|\sum_{1}^{N} b_{n} f\left(\gamma+\gamma_{n}\right)\left(y, \gamma+\gamma_{n}\right)\right|, \quad \gamma \in \Lambda_{0},
$$

is majorized by a certain function $F$ such that

$$
\int_{\Lambda_{0}} F(\gamma) d \gamma \leqq \nu\left(\Lambda_{0}\right)\left(\|\hat{f}\|_{\infty}+\varepsilon\right)
$$

where $\nu$ is Haar measure on $\Gamma$.

Proof. Extend $\Lambda_{0}$ to a compact neighborhood $\Lambda_{1}$ of 0 in $\Gamma$ and let $\Gamma_{1}$ be the locally compact group generated by $\Lambda_{1}$. Let $H_{1}$ be the annihilator of $\Gamma_{1}$ and put $G_{1}=G / H_{1}$. Then $G_{1}$ is the dual of $\Gamma_{1}$. By the structure theorem for compactly generated groups, (see e.g. [6, (9.8)]), $G_{1}$ is of the form $G_{1}=R^{a} \times T^{b} \times D$ where $a, b$ are nonnegative integers and $D$ a discrete group.

Choose a compact symmetric neighborhood $V_{0}$ of 0 in $G_{1}$, which is of the form described in Lemma 1 , in such a way that, if $u$ is any function on $G_{1}$ satisfying conditions (1), (2), (3) of Lemma 1, then

$$
\left|\int_{G_{1}} u(x)(x, \gamma) \hat{\varphi}(x) d x-\hat{\varphi}(0)\right|<\varepsilon \quad \text { for all } \gamma \in \Lambda_{0} \text { (compact). }
$$

Observe that, since $\varphi$ is concentrated on $\Gamma_{1} ; \hat{\varphi}$ is constant on the cosets of $H_{1}$ and therefore $\hat{\varphi}$ is defined on $G_{1}$.

Next choose $k \in L^{1}\left(\Gamma_{1}\right)$ such that $\hat{k}$ has compact support, say $K$, in $G_{1}$ and such that

$$
\begin{gathered}
\int_{\Gamma_{1}}|\varphi * k-\varphi|<\varepsilon m_{1}\left(V_{0}\right) \leqq \varepsilon, \quad V_{0} \text { small, } \\
\int_{\Gamma_{1}}|f * k-f|<\varepsilon m_{1}\left(V_{0}\right) \leqq \varepsilon
\end{gathered}
$$

where $m_{1}$ is Haar measure on $G_{1}$. By (10)

$$
\|\hat{\varphi} \hat{k}-\hat{\varphi}\|_{\infty}<\varepsilon \quad\left(\text { sup over } G_{1}\right) \text {. }
$$

Now $V_{0}$ and the compact set $K$ (which we may extend to include $V_{0}+V_{0}$ ) are fixed and we choose $u$ satisfying the six conditions (1)-(6) of Lemma 1 .

Then, by the $L^{1}$-inversion theorem, we have, for $\gamma \in \Gamma_{1}$,

$$
\begin{aligned}
\sum_{1}^{\infty} b_{n}(\varphi * k)\left(\gamma+\gamma_{n}\right) & =\int_{K} \hat{\varphi} \hat{k}(x) \sum_{1}^{\infty} b_{n}\left(x, \gamma+\gamma_{n}\right) d x \\
& =\int_{K}(\hat{\varphi} \hat{k})(x)(x, \gamma) u(x) d x .
\end{aligned}
$$


Hence, for large $N$ and any $\gamma \in \Gamma_{1}$,

$$
\left|\sum_{1}^{N} b_{n}(\varphi * k)\left(\gamma+\gamma_{n}\right)-\int_{K}(\hat{\varphi} \hat{k})(x)(x, \gamma) u(x) d x\right|<\varepsilon .
$$

By $\left(10^{\prime}\right)$ and (1)-(3),

$$
\left|\int_{K}(\hat{\varphi} \hat{k})(x)(x, \gamma) u(x) d x-\int_{K} \hat{\varphi}(x)(x, \gamma) u(x) d x\right|<\varepsilon .
$$

Whence, by (12) and (9),

We conclude

$$
\left|\sum_{1}^{N} b_{n}(\varphi * k)\left(\gamma+\gamma_{n}\right)-\hat{\varphi}(0)\right|<3 \varepsilon \text { for } \gamma \in \Lambda_{0} .
$$

$$
\int_{\Lambda_{0}}\left|\sum_{1}^{N} b_{n}(\varphi * k)\left(\gamma+\gamma_{n}\right)-\hat{\varphi}(0)\right| d \gamma<3 \varepsilon \nu\left(\Lambda_{0}\right) .
$$

(Since $\Gamma_{1}$ is open in $\Gamma$ we may take Haar measure on $\Gamma_{1}$ to be the restriction of Haar measure $\nu$ on $\Gamma$.)

Finally, by (10)

$$
\begin{aligned}
\int_{\Lambda_{0}}\left|\sum_{1}^{N} b_{n} \varphi\left(\gamma+\gamma_{n}\right)-\hat{\varphi}(0)\right| d \gamma & \leqq \sum_{1}^{N}\left|b_{n}\right| \varepsilon m_{1}\left(V_{0}\right)+3 \varepsilon \nu\left(\Lambda_{0}\right) \\
& \leqq \varepsilon+3 \varepsilon v\left(\Lambda_{0}\right),
\end{aligned}
$$

which is the property (7) required for $\varphi$.

Again, by the $L^{1}$-inversion theorem, we have, for any $y \in G_{1}$ and any $\gamma \in \Gamma_{1}$,

$$
\begin{aligned}
\left|\sum_{1}^{\infty} b_{n}(f * k)\left(\gamma+\gamma_{n}\right)\left(y, \gamma+\gamma_{n}\right)\right| & =\left|\int_{K}(\hat{f} \hat{k})(x) \sum b_{n}\left(x+y, \gamma+\gamma_{n}\right)\right| \\
& \leqq\|\hat{f} \hat{k}\|_{\infty} \int_{K+y}\left|\sum b_{n}\left(x, \gamma+\gamma_{n}\right)\right| \\
& \leqq\|\hat{f} \hat{k}\|_{\infty} \leqq\|\hat{f}\|_{\infty}+\varepsilon .
\end{aligned}
$$

Hence, for large $N$, and any $y \in G_{1}, \gamma \in \Gamma_{1}$,

$$
\left|\sum_{1}^{N} b_{n}(f * k)\left(\gamma+\gamma_{n}\right)\left(y, \gamma+\gamma_{n}\right)\right| \leqq\|\hat{f}\|_{\infty}+2 \varepsilon .
$$

We deduce, for $\gamma \in \Gamma_{1}$, since $\gamma_{n} \in \Gamma_{1}$ and the characters $\left(y, \gamma+\gamma_{n}\right)$ are constant on the cosets of $H_{1}$ :

$$
\begin{aligned}
\sup _{y \in G}\left|\sum_{1}^{N} b_{n} f\left(\gamma+\gamma_{n}\right)\left(y, \gamma+\gamma_{n}\right)\right| & =\sup _{y \in G_{1}}\left|\sum_{1}^{N} b_{n} f\left(\gamma+\gamma_{n}\right)\left(y, \gamma+\gamma_{n}\right)\right| \\
& \leqq \sup _{y \in G_{1}}\left|\sum_{1}^{N} b_{n}(f-f * k)\left(\gamma+\gamma_{n}\right)\left(y, \gamma+\gamma_{n}\right)\right|+\|\hat{f}\|_{\infty}+2 \varepsilon \\
& \leqq \sum_{1}^{N}\left|b_{n}(f-f * k)\left(\gamma+\gamma_{n}\right)\right|+\|\hat{f}\|_{\infty}+2 \varepsilon \\
& =F(\gamma) \text { say }
\end{aligned}
$$


where by (11)

$$
\begin{aligned}
\int_{\Lambda_{0}} F(\gamma) d \gamma & \leqq \sum_{1}^{N}\left|b_{n}\right| \varepsilon m_{1}\left(V_{0}\right)+\nu\left(\Lambda_{0}\right)\left(\|\hat{f}\|_{\infty}+2 \varepsilon\right) \\
& \leqq \varepsilon+\nu\left(\Lambda_{0}\right)\left(\|\hat{f}\|_{\infty}+2 \varepsilon\right) .
\end{aligned}
$$

$\varepsilon$ being arbitrary, (8) is now proved.

LEMMA 3. Assume $\varphi$ is measurable on $\Lambda \subset \Gamma, \varphi$ is zero outside $\Lambda$ and $\varphi$ is approximable on finite sets in $\Lambda$ with capacity at most $C$. For any $f$, bounded, measurable, vanishing outside $\Lambda$, with compact support, put $T(f)=\int_{\Gamma} f \varphi d \gamma$. Then $|T(f)| \leqq C\|\hat{f}\|_{\infty}$.

Proof. Let $\varepsilon>0$ be given and let $\Lambda_{0}$ be the compact support of $f$. By Lemma 2, applied to the two functions $f \varphi$ and $f$, both with compact support $\Lambda_{0}$, there is a polynomial $p(x)=\sum_{1}^{N} b_{n}\left(x, \gamma_{n}\right)$ such that

$$
\int_{\Lambda_{0}}\left|\sum_{1}^{N} b_{n} f\left(\gamma+\gamma_{n}\right) \varphi\left(\gamma+\gamma_{n}\right)-\int f \varphi\right| d \gamma<\varepsilon^{2} \nu\left(\Lambda_{0}\right)
$$

and

$$
\sup _{y}\left|\sum_{1}^{N} b_{n} f\left(\gamma+\gamma_{n}\right)\left(y, \gamma+\gamma_{n}\right)\right| \leqq F(\gamma), \quad \gamma \in \Lambda_{0},
$$

where

$$
\int_{\Lambda_{0}} F(\gamma) d \gamma \leqq \nu\left(\Lambda_{0}\right)\left(\|\hat{f}\|_{\infty}+\varepsilon\right)
$$

Put

$$
E_{1}=\left\{\gamma \in \Lambda_{0}:\left|\sum_{1}^{N} b_{n} f\left(\gamma+\gamma_{n}\right) \varphi\left(\gamma+\gamma_{n}\right)-\int f \varphi\right|>\varepsilon\right\} .
$$

Then, by (1)

$$
\varepsilon \cdot \nu\left(E_{1}\right)<\varepsilon^{2} \nu\left(\Lambda_{0}\right) ; \quad \nu\left(E_{1}\right)<\varepsilon \nu\left(\Lambda_{0}\right)
$$

Put

$$
E_{2}=\left\{\gamma \in \Lambda_{0}: F(\gamma)>(1-\varepsilon)^{-1}\left(\|\hat{f}\|_{\infty}+\varepsilon\right)\right\}
$$

By (2)

$$
\begin{aligned}
(1-\varepsilon)^{-1}\left(\|\hat{f}\|_{\infty}+\varepsilon\right) \nu\left(E_{2}\right) & \leqq \nu\left(\Lambda_{0}\right)\left(\|\hat{f}\|_{\infty}+\varepsilon\right), \\
\nu\left(E_{2}\right) & \leqq(1-\varepsilon) \nu\left(\Lambda_{0}\right) .
\end{aligned}
$$

We conclude $\nu\left(E_{1} \cup E_{2}\right)<\nu\left(\Lambda_{0}\right)$. Hence there is $\lambda_{0} \in \Lambda_{0}$ such that $\lambda_{0} \notin E_{1}, \lambda_{0} \notin E_{2}$, that is

$$
\begin{gathered}
\left|\sum_{1}^{N} b_{n} f\left(\lambda_{0}+\gamma_{n}\right) \varphi\left(\lambda_{0}+\gamma_{n}\right)-T(f)\right| \leqq \varepsilon, \\
\sup _{y}\left|\sum_{1}^{N} b_{n} f\left(\lambda_{0}+\gamma_{n}\right)\left(y, \lambda_{0}+\gamma_{n}\right)\right| \leqq(1-\varepsilon)^{-1}\left(\|\hat{f}\|_{\infty}+\varepsilon\right) .
\end{gathered}
$$


Let $A$ be the finite set of elements of the form $\lambda_{0}+\gamma_{n}, n=1, \ldots, N$, which belong to $\Lambda_{0} \cap \Lambda$. By hypothesis there is a polynomial $q(\gamma)=\sum_{m} c_{m}\left(y_{m}, \gamma\right)$ with $\sum\left|c_{m}\right| \leqq C$ such that

$$
|q(\gamma)-\varphi(\gamma)|<\varepsilon / \sum_{1}^{N}\left|b_{n}\right|\|f\|_{\infty} \text { for } \gamma \in A .
$$

Observing that $f\left(\lambda_{0}+\gamma_{n}\right)=0$ if $\lambda_{0}+\gamma_{n} \notin A$ we get from (3) and (5)

This is

$$
\left|T(f)-\sum_{1}^{N} b_{n} f\left(\lambda_{0}+\gamma_{n}\right) q\left(\lambda_{0}+\gamma_{n}\right)\right| \leqq \varepsilon+\varepsilon .
$$

$$
\left|T(f)-\sum_{m} c_{m} \sum_{n=1}^{N} b_{n} f\left(\lambda_{0}+\gamma_{n}\right)\left(y_{m}, \lambda_{0}+\gamma_{n}\right)\right| \leqq 2 \varepsilon .
$$

By (4) the coefficient of $c_{m}$ has modulus $\leqq(1-\varepsilon)^{-1}\left(\|\hat{f}\|_{\infty}+\varepsilon\right)$. Hence

$$
\begin{aligned}
|T(f)| & \leqq \sum_{m}\left|c_{m}\right|(1-\varepsilon)^{-1}\left(\|\hat{f}\|_{\infty}+\varepsilon\right)+2 \varepsilon \\
& \leqq C(1-\varepsilon)^{-1}\left(\|\hat{f}\|_{\infty}+\varepsilon\right)+2 \varepsilon .
\end{aligned}
$$

Since $\varepsilon$ is arbitrary, the lemma is proved.

MAIN THEOREM. Assume that $\varphi$ is measurable on the measurable set $\Lambda$ and that $\varphi$ is approximable on finite sets in $\Lambda$ with capacity at most $C$. Then $\varphi(\gamma)=\hat{\mu}(\gamma)$ locally almost everywhere on $\Lambda$, where $\mu \in M(G)$ and $\|\mu\| \leqq C$.

Proof. The linear functional $S$ given by $S(\hat{f})=T(f)=\int_{\Gamma} f \varphi d \gamma$ is defined on the linear space of the transforms $\hat{f}$ of the bounded measurable functions $f$ vanishing outside $\Lambda$, with compact support (a subspace of $C_{0}(G)$ ) and satisfies the inequality $|S(\hat{f})| \leqq C\|\hat{f}\|_{\infty}$.

By the Hahn-Banach theorem, $S$ can be extended to the whole of $C_{0}(G)$, with norm not exceeding $C$. By the Riesz-Kakutani representation theorem there is a $\mu \in M(G)$ such that $\|\mu\| \leqq C$ and $S(\hat{f})=\int_{G} \hat{f}(x) d \mu(x)$. Then, by Fubini's theorem

$$
S(\hat{f})=\int_{\Gamma} f(\gamma) \hat{\mu}(\gamma) d \gamma
$$

that is, $\int_{\Gamma} f(\gamma) \varphi(\gamma) d \gamma=\int_{\Gamma} f(\gamma) \hat{\mu}(\gamma) d \gamma$ for every $f$, bounded, vanishing outside $\Lambda$, with compact support. We conclude $\varphi(\gamma)=\hat{\mu}(\gamma)$ locally a.e. on $\Lambda$ and the theorem is proved.

REMARK. This theorem contrasts with the situation where instead of the transform of a measure we consider positive definite functions (the transforms of positive measures).

Suppose $\Lambda_{0}$ is a measurable subset of a locally compact abelian group $\Gamma$. Define $P D\left(\Lambda_{0}\right)$ to be the class of all continuous complex valued functions $\varphi$ on $\Lambda_{0}-\Lambda_{0}$ which satisfy the inequality

$$
\sum_{i, j=1}^{N} c_{i} \bar{c}_{j} \varphi\left(\gamma_{i}-\gamma_{j}\right) \geqq 0
$$


for every positive integer $N$, for every choice of complex numbers $c_{1}, \ldots, c_{N}$ and for every choice of points $\gamma_{1}, \ldots, \gamma_{N}$ in $\Lambda_{0}$.

If $G=R, \Lambda_{0}=$ an interval $I$, then every $\varphi \in P D(I)$ is the restriction on $I-I$ of the transform $\hat{\mu}$ of some positive measure $\mu$ on $G$ (Krein). But if $G=R^{2}, \Lambda_{0}=$ a closed square $S$ in $R^{2}$, then there is $\varphi \in P D(S)$ which is not the restriction to $S-S$ of the transform of a positive measure on $G$ (see Rudin [8]).

Before ending our paper we want to state, in a new form, the two theorems appearing in [4].

TheOrem. A continuous function $\varphi$ defined on $\Gamma$ is the Fourier-Stieltjes transform of a singular measure on $G$ if and only if there is a constant $C$ such that

(i) $\varphi$ can be approximated on any finite set in $\Gamma$ by trigonometric polynomials of capacity at most $C$.

(ii s) Whatever be $\varepsilon>0$ and the compact set $\Lambda$ in $\Gamma, \varphi$ is not approximable on finite sets $F$, not meeting $\Lambda$, by polynomials of capacity $\leqq C-\varepsilon$.

TheOREM. A continuous function $\varphi$ defined on $\Gamma$ is the Fourier transform of an integrable function on $G$ if and only if

(i) there is a constant $C$ such that $\varphi$ can be approximated on any finite set in $\Gamma$ by trigonometric polynomials of capacity at most $C$.

(ii a) To every $\varepsilon>0$ corresponds a compact set $\Lambda$ in $\Gamma$ such that $\varphi$ can be approximated on any finite set in $\Gamma$, not meeting $\Lambda$, by trigonometric polynomials of capacity at most $\varepsilon$.

To prove these theorems we just make use of the equivalence stated in Proposition 2 and combine this equivalence with Theorems 1 and 2 of [4]. Observe also that no form of any of these two theorems is readily available for restrictions, since the transform $\hat{\mu}_{s}$ of a singular measure can be equal to the transform $\hat{f}$ of an integrable function on very" large sets (see e.g. Rudin [9]).

\section{BIBLIOGRAPHY}

1. N. I. Ahiezer, Lectures on the theory of approximation, OGIZ, Moscow, 1947; English transl., Ungar, New York, 1956. MR 10, 33; MR 20 \#1872.

2. S. Bochner, A theorem on Fourier-Stieltjes integrals, Bull. Amer. Math. Soc. 40 (1934), 271-276.

3. K. de Leeuw and C. Herz, An invariance property of spectral synthesis, Illinois J. Math. 9 (1965), 220-229. MR 31 \#3787.

4. R. Doss, On the transform of a singular or an absolutely continuous measure, Proc. Amer. Math. Soc. 19 (1968), 361-363. MR 36 \#5619.

5. W. F. Eberlein, Characterizations of Fourier-Stieltjes transforms, Duke Math. J. 22 (1955), 465-468. MR 17, 281.

6. E. Hewitt and K. A. Ross, Abstract harmonic analysis. Vol. I: Structure of topological groups. Integration theory, group representations, Die Grundlehren der math. Wissenschaften, Band 115, Academic Press, New York, and Springer-Verlag, Berlin, 1963. MR 28 \#158. 
7. H. P. Rosenthal, A characterization of restrictions of Fourier-Stieltjes transforms, Pacific J. Math. 23 (1967), 403-418. MR 36 \#3065.

8. W. Rudin, The extension problem for positive-definite functions, Illinois J. of Math. 7 (1963), 532-539. MR 27 \#1779.

9. _ Modifications of Fourier transforms, Proc. Amer. Math. Soc. 19 (1968), 10691074. MR 39 \#1903.

State University OF NeW York,

STONY BROOK, NEW YoRK 11790 